\title{
Happiness by Design: A Self-Construction Framework of Proteus and Networking Effects in Online Games: An Abstract
}

\author{
Ting-Ting Chen, Shih-Ju Wang, Huang Heng-Chiang, and Shih-Tsen Wang
}

\begin{abstract}
The emergence of the digital world has provided users an online platform to alter their digital self-representations dramatically and easily. Today, mobile games, as entertainment media due to the rapid growth of mobile devices, usually become players' utopian "third place," beyond home and the workplace.

Adopting self-construal theory, we propose a causal model that incorporates both the interdependent-self and independent-self routes, which lead to players' online game addiction and perceived psychological well-being. To test the model using SmartPLS 3.2, we recruited 242 players of the Tower of Virtual Saviors mobile game in Taiwan.

Both the interdependent-self route (from knowledge sharing to network externality, to social capital, to sense of virtual community) and the independent-self route (from social anxiety to disinhibition) exert a positive influence on players' game addiction, which ultimately strengthens their psychological well-being. Theoretical and marketing implications of the findings are discussed.
\end{abstract}

References Available Upon Request

T.-T. Chen $(\bowtie) \bullet$ H. Heng-Chiang $\bullet$ S.-T. Wang

National Taiwan University, Taipei City, Taiwan

e-mail: smilestop1213@gmail.com; huang@ntu.edu.tw; pandaiseddie@gmail.com

S.-J. Wang

National Taiwan Normal University, Taipei City, Taiwan

e-mail: sjwang@ntnu.edu.tw 